\title{
Mechanism Design for Fair and Efficient DSO Flexibility Markets
}

\author{
Tsaousoglou, Georgios; Giraldo, Juan S.; Pinson, Pierre; Paterakis, Nikolaos G.
}

\section{Published in:}

IEEE Transactions on Smart Grid

Link to article, DOI:

10.1109/TSG.2020.3048738

Publication date:

2021

Document Version

Peer reviewed version

Link back to DTU Orbit

Citation (APA):

Tsaousoglou, G., Giraldo, J. S., Pinson, P., \& Paterakis, N. G. (2021). Mechanism Design for Fair and Efficient DSO Flexibility Markets. IEEE Transactions on Smart Grid, 12(3), 2249-2260. [9312120].

https://doi.org/10.1109/TSG.2020.3048738

\section{General rights}

Copyright and moral rights for the publications made accessible in the public portal are retained by the authors and/or other copyright owners and it is a condition of accessing publications that users recognise and abide by the legal requirements associated with these rights.

- Users may download and print one copy of any publication from the public portal for the purpose of private study or research.

- You may not further distribute the material or use it for any profit-making activity or commercial gain

- You may freely distribute the URL identifying the publication in the public portal 


\title{
Mechanism Design for Fair and Efficient DSO Flexibility Markets
}

\author{
Georgios Tsaousoglou, Juan S. Giraldo, Member, IEEE, Pierre Pinson, Fellow, IEEE, \\ and Nikolaos G. Paterakis, Member, IEEE
}

\begin{abstract}
The proliferation of distributed energy assets necessitates the provision of flexibility to efficiently operate modern distribution systems. In this paper, we propose a flexibility market through which the DSO may acquire flexibility services from asset aggregators in order to maintain network voltages and currents within safe limits. A max-min fair formulation is proposed for the allocation of flexibility. Since the DSO is not aware of each aggregator's local flexibility costs, we show that strategic misreporting can lead to severe loss of efficiency. Using mechanism design theory, we provide a mechanism that makes it a payoff-maximizing strategy for each aggregator to make truthful bids to the flexibility market. While typical truthful mechanisms only work when the objective is the maximization of Social Welfare, the proposed mechanism lets the DSO achieve incentive compatibility and optimality for the the max-min fairness objective.
\end{abstract}

Index Terms-Flexibility, Distribution System, Incentive Compatibility, Mechanism Design, Fairness, Aggregator

\section{NOMENCLATURE}

Sets

$\Omega_{b} \quad$ Set of nodes / aggregators.

$\Omega_{l} \quad$ Set of lines.

$\Omega_{\mathrm{T}} \quad$ Set of time periods.

\section{Parameters}

$\lambda_{i, t} \quad$ Parameter of flexibility cost at node $i$, period $t$ $\left[\$ / \mathrm{kWh}^{2}\right]$.

$\overline{\mathrm{K}}_{i, t} \quad$ Maximum flexibility factor at node $i$, period $t$.

$\underline{\mathrm{K}}_{i, t}$ Minimum flexibility factor at node $i$, period $t$.

$\overline{\mathrm{I}}_{i j} \quad$ Maximum current magnitude for line $i j[\mathrm{~A}]$.

$\mathrm{P}_{i, t}^{\mathrm{D}} \quad$ Active power demand at node $i$, period $t[\mathrm{~kW}]$.

$\mathrm{Q}_{i, t}^{\mathrm{D}} \quad$ Reactive power demand at node $i$, period $t$ [kvar].

$\mathrm{R}_{\mathrm{ij}} \quad$ Resistance of line $i j[\mathrm{~m} \Omega]$.

$\overline{\mathrm{V}} \quad$ Maximum voltage magnitude $[\mathrm{kV}]$.

$\underline{\mathrm{V}} \quad$ Minimum voltage magnitude $[\mathrm{kV}]$.

$\mathrm{X}_{\mathrm{ij}} \quad$ Reactance of line $i j[\mathrm{~m} \Omega]$.

Variables

$K_{i, t} \quad$ Flexibility factor at node $i$, period $t$.

$I_{i j, t}^{\mathrm{sqr}} \quad$ Squared current magnitude at line $i j$, period $t\left[\mathrm{~A}^{2}\right]$.

$P_{i j, t} \quad$ Active power flow at line $i j$, period $t[\mathrm{~kW}]$.

Georgios Tsaousoglou received funding from the European Union's Horizon 2020 research and innovation programme under the Marie Skłodowska-Curie grant agreement No.754462.

G. Tsaousoglou, J.S. Giraldo and N.G. Paterakis are with the Eindhoven University of Technology. P. Pinson is with the Technical University of Denmark.
$P_{i, t}^{\mathrm{S}} \quad$ Active power injection at substation $i$, period $t[\mathrm{~kW}]$.

$Q_{i j, t}$ Reactive power flow at line $i j$, period $t$ [kvar].

$Q_{i, t}^{\mathrm{S}} \quad$ Reactive power injection at substation $i$, period $t$ [kvar].

$V_{i, t}^{\text {sqr }}$ Squared voltage magnitude at node $i$, period $t\left[\mathrm{kV}^{2}\right]$.

\section{INTRODUCTION AND RELATED WORK}

A LONG with high penetration of renewable energy sources (RES), electricity distribution systems face several challenges relating to their safe and reliable operation. Flexible distributed resources are seen as an important asset towards accommodating the intermittent nature of RES generation. These flexibility assets may include small-scale generation, distributed storage facilities, building energy management systems, electric vehicles etc., and their flexibility refers to the ability of controlling their power profile.

Due to their small size and large numbers, distributed flexibility assets cannot be incorporated in the electricity markets because they would dramatically increase the number of decision variables and constraints in the operator's market clearing problem. Thus, market participation of small flexibility assets is envisioned via aggregators. An aggregator is an entity that provides flexibility services on behalf of the flexible assets in its portfolio [1], [2]. The framework for flexibility aggregation is not trivial, and has been the topic of recent studies (e.g. [3]).

In modern electricity systems, buyers and sellers freely trade energy through electricity pool markets or through bilateral contracts. However, at a certain time before actual delivery (the gate closure time), the trading stops and the system operator makes sure that the physical constraints of the underlying grid are respected. The energy transactions of a player until the gate closure time, constitute the player's market program. The players' market programs may not be feasible for the physical distribution system to support. Thus, distribution system operators (DSOs) need to use flexibility services provided locally by aggregators in order to keep the network within safe operational limits.

In this paper we consider a distribution system and a set of aggregators, where each aggregator represents a set of loads along with a set of flexibility assets. The second-order cone programming relaxation based on the branch flow model as in [4], [5] has been used to represent the operation of the distribution system. Given the day-ahead program, the DSO calls on the aggregators' flexibility in order to satisfy the physical network's constraints. An aggregator provides 
flexibility by modifying its demand profile with respect to its day-ahead market program.

Depending on the type of assets that constitute the aggregator's portfolio, this modification can be realized in different ways. For example, electric vehicle aggregators can shift charging to later timeslots [6] or coordinate the geographical distribution of vehicles via incentives and prices [7]. In similar fashion, residential aggregators and building management systems can control the smart appliances of buildings (e.g., by manipulating the thermostats of thermostatically controlled loads). These services come at the cost of possibly causing discomfort to the end users, which is why aggregators compensate the users for their flexibility provision via data-driven methods [8], dynamic pricing schemes [9] or transactive local energy markets [10].

Based on the above, flexibility comes at a financial cost that the aggregator has to pay to its flexibility assets in order to incentivize them to provide the requested service. Thus, each aggregator bids a flexibility cost function to the DSO's flexibility market. The peculiarity of a DSO flexibility market is that, due to the physical network's topology, it is very often the case that the flexibility of a particular node is necessary for the safe network operation. This means that an aggregator can strategically inflate its bid in order to benefit from price manipulation. Furthermore, aggregators may also decide to not follow the DSO instruction if they find that to be of benefit for them. The potential of strategic behaviour by aggregators has been demonstrated in recent studies [11], [12]. In [13], it was also shown that even small aggregators can manipulate the price by leveraging their grid location. Moreover, in [14], deep reinforcement learning is shown to be a well-performing method for aggregators to compute such a strategy.

For these reasons, it is important to implement a market mechanism where aggregators are incentivized to make a truthful report on their flexibility costs and have nothing to gain by inflating their bids or by not following the DSO instructions. A mechanism is defined by an allocation rule, i.e., the way that resources are being allocated to participating agents, and a payment rule, i.e., the way that agent paymets/rewards are being calculated.

In the literature of electricity systems, the allocation rule is typically defined by solving an optimization problem that maximizes social welfare, while the payment rule is defined by calculating a set of Lagrange multipliers, where the Lagrange multiplier of a node's power balance constraint is interpreted as the node's locational price. This concept has already been proposed also for DSO markets, and is usually referred to as Distribution Locational Marginal Pricing, e.g., [15], [16], [17], [18], [19]. It is important to note that the Lagrangian methods [15]- [19] do not provide protection against strategic behavior and they are vulnerable to price manipulation by strategic participants. One way to deal with strategic behavior is to employ ex-post market monitoring techniques as proposed in [20], while another is to ex-ante employ an incentive compatible payment mechanism.

A mechanism that makes truthful reporting a profitmaximizing strategy is said to have the property of incentive compatibility. In more detail, there are different notions of incentive compatibility, the strongest of which is called Dominant Strategy Incentive Compatibility (or DSIC). We say that a mechanism is DSIC when the best (dominant) strategy of every participating player is to be truthful, regardless of what other players do.

Some studies in the area of the smart gird have leveraged concepts from mechanism design to design payment rules for various use cases. In [21], a payment rule is designed, to incentivize residential load agents to truthfully report their expected demand to a load aggregator. In [22], a payment rule is designed so that flexible electricity assets are incentivized to shift their consumption away from peak-demand timeslots. In [23], an asymptotically truthful rule (not DSIC) is designed to allocate electricity consumption to a set of flexible household agents under certain assumptions on the agents' local valuation functions.

Another family of studies in the smart gird literature, leverages the (DSIC and optimal) Vickrey-Clarke-Groves (VCG) mechanism for achieving truthfulness and optimality. Examples include [24] and [25], while in [26], the authors propose a distributed implementation of VCG to achieve truthfulness along with scalability and privacy in a Demand Response scheme.

Since the studies mentioned above ( [23]- [26]), examine different use cases, each one proposes a different payment rule. However, the underlying objective in all of them is social welfare maximization, which, although formulated differently in different use cases, means that the allocation rules are similar in nature: minimizing the average agent cost.

In the setting of a DSO flexibility market, however, the typical approach of minimizing the average flexibility cost among agents can have detrimental effects on the payoff of some particular participant, i.e., the mechanism may "sacrifice" the payoff of a certain node or aggregator, in order to benefit the whole system. Especially in a distribution system, the grid topology might cause a particular set of users (that are sited under a particular node of the grid) to be repeatedly treated unfairly. Such phenomena can lead to non-sustainable business models for some aggregators, which can result in market concentration and deteriorate market competition.

In order to prevent such phenomena, the objective of the market can be defined so as to maximize fairness. In [27] and [28], fairness is defined using the Shapley value, whereas in [29] a compensation mechanism for flexibility activation is proposed as a fair way to compensate aggregators. In [30] and [31] fairness is assessed on the basis of equally distributing electricity costs among users, based on their level of effect on the community's electricity cost. However, in order to remedy issues of aggregators sustainability and thus promote market competition, the most relevant objective is to maximize the lowest payoff among aggregators. This is commonly referred to as optimizing for max-min fairness and is generally considered a fairness criterion in various fields (e.g. loadbalancing in communication networks [32]). In [33], max-min fairness is assessed for a DR setting with electric vehicles and cooling devices, while in [34], max-min fairness optimization is considered and solved using the alternating direction method of multipliers (ADMM) algorithm. In contrast to the case of 
minimizing the average cost, optimizing for max-min fairness is not thoroughly studied in the smart grid literature. Moreover, the optimal and incentive compatible VCG mechanism only works when the objective maximizes the social welfare and not fairness.

In fact, to the best of our knowledge, developing a flexibility mechanism that is simultaneously fair and incentive compatible has not been dealt with before. In this paper we consider a distribution system, where the DSO calls on the aggregators' flexibility in order to satisfy the physical constraints of the system. We design a DSIC mechanism that incentivizes aggregators to declare their true cost for flexibility provision at the scheduling phase, and then follow the DSO's instruction at real-time operation. Thus, the main contributions of this paper can be summarized as follows:

- We formulate a max-min fair allocation problem for flexibility in a distribution system, so that network constraints are satisfied in a fair way.

- We design an incentive compatible mechanism so that flexibility aggregators are incentivized to declare their true cost for flexibility at the scheduling phase, and then follow the instructions of the DSO at real-time operation.

- We thoroughly evaluate the proposed system through simulations and compare it to different benchmarks.

The remainder of this paper is organized as follows: Section II presents the system model and the power flow equations of the distribution network. In Section III the problem formulation is presented and in Section IV the proposed reward function is built, and its properties are theoretically proven. In Section V, simulation results for various test cases are presented and Section VI concludes the paper.

\section{Distribution System Model}

We consider a distribution system, constituted by a set $\Omega_{b}$ of nodes $b \in \Omega_{b}$ and a set $\Omega_{l}$ of lines $l \in \Omega_{l}$. We consider a set of aggregators where each aggregator is responsible for the net demand of its portfolio. We assume that the DSO has access to measurement facilities and can observe each aggregator's power consumption. In order to facilitate the analysis, we assume that each node of the distribution network is represented by one demand aggregator $i \in \Omega_{b}$, however the methods and results of this paper hold equivalently for the general case. For a horizon of discrete timeslots $t \in \Omega_{\mathrm{T}}$, an aggregator $i \in \Omega_{b}$ is characterized by an active power demand profile $\mathbf{P}_{i}^{\mathrm{D}}=\left\{\mathrm{P}_{i, t}^{\mathrm{D}}, t \in \Omega_{\mathrm{T}}\right\}$ constituted by the aggregator's demand at each timeslot. The profile $\mathbf{P}_{i}^{\mathrm{D}}$ represents the energy bought in the day-ahead market and it is communicated by the day-ahead market operator to the DSO.

The DSO is responsible for the operation of the distribution system, so that voltage and current magnitudes remain within safe limits. For this purpose, the DSO draws on the flexibility offered by the aggregators in $\Omega_{b}$, that is, the ability of an aggregator to modify its active power demand $\mathrm{P}_{i, t}^{\mathrm{D}}$ at timeslot $t$ by a certain factor $K_{i, t}$. A modification $K_{i, t} \mathrm{P}_{i, t}^{\mathrm{D}}$, results in an active power injection $P_{i, t}^{\mathrm{S}}$, determined by the active power balance equation

$$
\begin{array}{r}
\sum_{k i \in \Omega_{l}} P_{k i, t}-\sum_{i j \in \Omega_{l}}\left(P_{i j, t}+\mathrm{R}_{i j} I_{i j, t}^{\mathrm{sqr}}\right)+P_{i, t}^{\mathrm{S}}=K_{i, t} \mathrm{P}_{i, t}^{\mathrm{D}}, \\
\forall i \in \Omega_{b}, t \in \Omega_{\mathrm{T}}
\end{array}
$$

The corresponding reactive power flows are represented as

$$
\begin{array}{r}
\sum_{k i \in \Omega_{l}} Q_{k i, t}-\sum_{i j \in \Omega_{l}}\left(Q_{i j, t}+\mathrm{X}_{i j} I_{i j, t}^{\mathrm{sqr}}\right)+Q_{i, t}^{\mathrm{S}}=K_{i, t} \mathrm{Q}_{i, t}^{\mathrm{D}}, \\
\forall i \in \Omega_{b}, t \in \Omega_{\mathrm{T}}
\end{array}
$$

Notice that aggregators are assumed to maintain a constant power factor and also, it is assumed $P_{i, t}^{\mathrm{S}}=Q_{i, t}^{\mathrm{S}}=0$ for all buses but the substation. On the other hand, for the substation bus, notice that $P_{0, t}^{\mathrm{S}}$ can also be less than 0 , representing backfeeding. It should be noted that these assumptions are without loss of generality with respect to the methods that will be presented.

Since current and voltage magnitudes appear naturally as squared, non-negative, continuous variables in the steady-state operation of AC electrical distribution systems, it is convenient to perform the change in variables $V_{i, t}^{\mathrm{sqr}} \equiv V_{i, t}^{2}$ and $I_{i j, t}^{\mathrm{sqr}} \equiv I_{i j, t}^{2}$ as in [35]. The voltage magnitude drop between nodes $i$ and $j$ is represented by

$$
\begin{array}{r}
V_{i, t}^{\mathrm{sqr}}-2\left(\mathrm{R}_{i j} P_{i j, t}+\mathrm{X}_{i j} Q_{i j, t}\right)-\left(\mathrm{R}_{i j}^{2}+\mathrm{X}_{i j}^{2}\right) I_{i j, t}^{\mathrm{sqr}} \\
=V_{j, t}^{\mathrm{sqr}}, \\
\forall i j \in \Omega_{l}, t \in \Omega_{\mathrm{T}}
\end{array}
$$

while current magnitudes are calculated using

$$
V_{j, t}^{\mathrm{sqr}} I_{i j, t}^{\mathrm{sqr}}=P_{i j, t}^{2}+Q_{i j, t}^{2}, \quad \forall i j \in \Omega_{l}, t \in \Omega_{\mathrm{T}}
$$

Upper and lower bounds on nodal voltage magnitudes and current magnitudes are enforced by

$$
\begin{array}{lr}
\underline{\mathrm{V}}^{2} \leq V_{i, t}^{\mathrm{sqr}} \leq \overline{\mathrm{V}}^{2} & \forall i \in \Omega_{b}, t \in \Omega_{\mathrm{T}} \\
0 \leq I_{i j, t}^{\mathrm{sqr}} \leq \overline{\mathrm{I}}_{i j}^{2} & \forall i j \in \Omega_{l}, t \in \Omega_{\mathrm{T}}
\end{array}
$$

An aggregator's flexibility is bounded as in

$$
\underline{\mathrm{K}}_{i, t} \leq K_{i, t} \leq \overline{\mathrm{K}}_{i, t} \quad \forall i \in \Omega_{b}, t \in \Omega_{\mathrm{T}}
$$

Let $\boldsymbol{K}_{i}=\left\{K_{i, t}, t \in \Omega_{\mathrm{T}}\right\}$ denote $i$ 's profile of flexibility activation. Accordingly, tuple $\boldsymbol{L}_{i}=\left\{\lambda_{i, t}, \underline{\mathrm{K}}_{i, t}, \overline{\mathrm{K}}_{i, t}, t \in \Omega_{\mathrm{T}}\right\}$ denotes the aggregator's local parameters, where $\lambda_{i, t}$ is a cost parameter for flexibility activation. It is assumed that the aggregator draws its flexibility by calling on demandresponse resources from the prosumers in its portfolio at a cost defined by function $c_{i}\left(\boldsymbol{K}_{i}, \boldsymbol{L}_{i}\right)$. This is modelled by causing a deviation in the prosumers' active power demand (from $\mathrm{P}_{i, t}^{\mathrm{D}}$ to $\left.\mathrm{P}_{i, t}^{\mathrm{D}}\left(1-K_{i, t}\right)\right)$, which comes at a cost defined as

$$
c_{i}\left(\boldsymbol{K}_{i}, \boldsymbol{L}_{i}\right)=\sum_{t \in \Omega_{\mathrm{T}}} \lambda_{i, t}\left(\mathrm{P}_{i, t}^{\mathrm{D}}\left(1-K_{i, t}\right)\right)^{2}, \quad \forall i \in \Omega_{b}
$$

\section{PRoblem Formulation}

The objective of the DSO is to satisfy the operational constraints of the distribution system, while minimizing the highest flexibility cost incurred among aggregators by deciding the optimal flexibility activation. In other words, the DSO pursues a fair allocation of flexibility costs among aggregators so that all aggregators are motivated to contribute, while no 
particular aggregator has to be compromised to unilaterally facilitate the DSO in achieving its objectives. This optimization problem can be formulated as

$$
\begin{gathered}
\min _{\boldsymbol{K}_{i}, V_{i, t}^{\mathrm{sqr}}, I_{i j, t}^{\mathrm{sqr}}, P_{i j, t}, Q_{i j, t}, P_{0, t}^{\mathrm{S}}, Q_{0, t}^{\mathrm{S}}} \max _{i \in \Omega_{b}}\left\{c_{i}\left(\boldsymbol{K}_{i}, \boldsymbol{L}_{i}\right)\right\} \\
\text { s.t (1)-(8) }
\end{gathered}
$$

By introducing the slack variable $z$, and replacing (4) with

$$
V_{j, t}^{\mathrm{sqr}} I_{i j, t}^{\mathrm{sqr}} \geq P_{i j, t}^{2}+Q_{i j, t}^{2}, \quad \forall i j \in \Omega_{l}, t \in \Omega_{\mathrm{T}}
$$

problem (9) can be recast as second order cone programming problem that is represented by

$$
\begin{aligned}
\min _{z, \boldsymbol{K}_{i}, V_{i, t}^{\mathrm{sqr}}, I_{i j, t}^{\mathrm{sqr}}, P_{i j, t}, Q_{i j, t}, P_{0, t}^{\mathrm{S}}, Q_{0, t}^{\mathrm{S}}}\{z\} \\
\text { s.t } \quad z \geq c_{i}\left(\boldsymbol{K}_{i}, \boldsymbol{L}_{i}\right), \quad \forall i \in \Omega_{b} \\
\text { (1)-(3), (5)-(8), and (10) }
\end{aligned}
$$

Notice that under certain conditions, constraint (4) is equivalent to (10) in radial distribution systems, as explained in [4] and [5].

The model in (11) could be efficiently tackled assuming that the DSO knows the flexibility cost parameters $\lambda_{i, t}$, as well as the limits $\underline{\mathrm{K}}_{i, t}$ and $\overline{\mathrm{K}}_{i, t}$ for each aggregator. However, these parameters depend on each aggregator's demand response capabilities based on the scheduling of the flexible loads in its portfolio. Thus, the DSO does not have direct access to this information and relies on the aggregator's self report. An important problem arises, concerning the aggregator's truthfulness upon declaring its local parameters. Naturally, unless the aggregator is compensated for the service provided, it would opt for maximizing its own revenue, which is done by declaring no available flexibility. Moreover, a compensation scheme that is not carefully designed, might still result in strategic misreporting.

Let $\widehat{\boldsymbol{L}}_{i}=\left\{\widehat{\lambda}_{i, t}, \widehat{\mathrm{K}}_{i, t}, \widehat{\overline{\mathrm{K}}}_{i, t}\right\}$ denote the aggregator's declaration for $\boldsymbol{L}_{i}$ which may or may not be equal to the actual $\boldsymbol{L}_{i}$. The DSO solves (11) using the declarations $\widehat{\boldsymbol{L}}_{i}$ as input, and the optimal solution is given to each aggregator as an instruction $\boldsymbol{K}_{i}^{*}$ to follow.

Once the instruction is communicated to each aggregator for the horizon $\Omega_{\mathrm{T}}$, real-time operation begins. The aggregator implements a flexibility profile $\widetilde{\boldsymbol{K}}_{i}$, where the tilde denotes the fact that this is the actual final flexibility factor implemented by the aggregator, as measured by the DSO's smart meters. In real time operation, two things may go differently than scheduled:

- The aggregator might not follow the instruction, and implement a different final profile, i.e., $\widetilde{\boldsymbol{K}}_{i} \neq \boldsymbol{K}_{i}^{*}$.

- The actual cost $\widetilde{c}_{i}=c_{i}\left(\widetilde{\boldsymbol{K}}_{i}, \boldsymbol{L}_{i}\right)$ that the aggregator pays to its customers for the implementation of the flexibility profile $\widetilde{\boldsymbol{K}}_{i}$, may not be equal to the cost calculated by the DSO, $c_{i}\left(\widetilde{\boldsymbol{K}}_{i}, \widehat{\boldsymbol{L}}_{i}\right)$, e.g., aggregators providing false declarations of flexibility costs.

Note that both cases result in inefficiency, since even after assuming that the aggregator perfectly follows the instruction, the costs can still differ, i.e., $\widetilde{c}_{i}=c_{i}\left(\boldsymbol{K}_{i}^{*}, \boldsymbol{L}_{i}\right) \neq c_{i}\left(\boldsymbol{K}_{i}^{*}, \widehat{\boldsymbol{L}}_{i}\right)$. The efficiency sensitivity to aggregator's declarations will be addressed in a later section.
The payoff of an aggregator $i$, defined as $\pi_{i}$, is calculated as the compensation $p_{i}$ received by the DSO for its flexibility services, minus the actual cost $\widetilde{c}_{i}$ paid to its flexible customers according to

$$
\pi_{i}=p_{i}-\widetilde{c}_{i}
$$

The task of the DSO in this case is to design the reward function, $p_{i}$, in such a way that it makes it a dominant strategy for each aggregator to first make truthful declarations and then follow the flexibility instruction, i.e., $\widehat{\boldsymbol{L}}_{i} \approx \boldsymbol{L}_{i}$ and $\widetilde{\boldsymbol{K}}_{i} \approx \boldsymbol{K}_{i}^{*}$, respectively. That is, the reward function $p(\cdot)$ should be incentive compatible in the sense that it aligns the aggregators' local objectives with the general objective of the DSO. This needs to be done in order to achieve the optimal solution to problem (11), since this solution guarantees the safe operation of the distribution system and fairness for flexibility assets.

A general overview of the proposed procedure and message exchange is depicted in Fig. 1. First, the DSO is informed about the aggregators' expected demand profiles $\mathbf{P}_{i}^{\mathrm{D}}$ for a given time horizon ahead. The DSO checks whether the network constraints can be satisfied. In case they cannot be satisfied, the DSO calls for the aggregators' flexibility bids. The aggregators make their flexibility bids $\widehat{\boldsymbol{L}}_{i}$ to the DSO. Given the bids, the DSO solves (11) and communicates the instruction $\boldsymbol{K}_{i}^{*}$ to each aggregator $i$. Given the instruction, the aggregator manages its portfolio at real time operation. The aggregator's actual load profile $\widetilde{\boldsymbol{K}}_{i}$ can be measured by the DSO. After the end of the horizon, the aggregator compensates its flexible agents and communicates the "proof of payment" to the DSO, i.e., the voucher $\widetilde{c}_{i}$. This could also be done automatically e.g. via a smart contract. Given the aggregator's instruction and observation (i.e. measured consumption and proof of payments), the DSO calculates the aggregator's compensation $p_{i}$.

\section{REWARD FUNCTION DESIGN}

For the design of the reward function $p_{i}$ we consider the family of mechanisms referred to as compensation and penalty mechanisms (CPM), as in [36], Section 10.6.1. In our context, the aggregators' instructions $\boldsymbol{K}_{i}^{*}$, are calculated by solving problem (11), but using the declared tuples $\widehat{\boldsymbol{L}}_{i}$ as the input that defines the functions $c_{i}\left(\boldsymbol{K}_{i}, \widehat{\boldsymbol{L}}_{i}\right)$. After the observation of the outcome, the aggregator's reward is calculated by

$$
p_{i}^{\mathrm{CPM}}=\widetilde{c}_{i}-\beta \max \left\{\widetilde{c}_{i}, \max _{j \in \Omega_{b}: j \neq i}\left\{c_{j}\left(\boldsymbol{K}_{j}^{*}, \widehat{\boldsymbol{L}}_{j}\right)\right\}\right\}
$$

As explained above, $\widetilde{c}_{i}=c_{i}\left(\widetilde{\boldsymbol{K}}_{i}, \boldsymbol{L}_{i}\right)$ is the observation of the actual flexibility costs for aggregator $i$ after the end of the horizon, while $\widetilde{\boldsymbol{K}}_{i}$ is the measured flexibility factor, which is calculated at the end of the horizon based on the measurements of the actual power injections. The first term in (13) compensates the aggregator for its actual flexibility cost that the latter offered at its flexible assets. The second term is the worst-off flexibility cost among aggregators, where the cost is calculated over the declared tuples $\widehat{\boldsymbol{L}}_{j}$ and instruction factors $\boldsymbol{K}_{j}^{*}$ of the rest of the aggregators, $j \neq i$, but over 


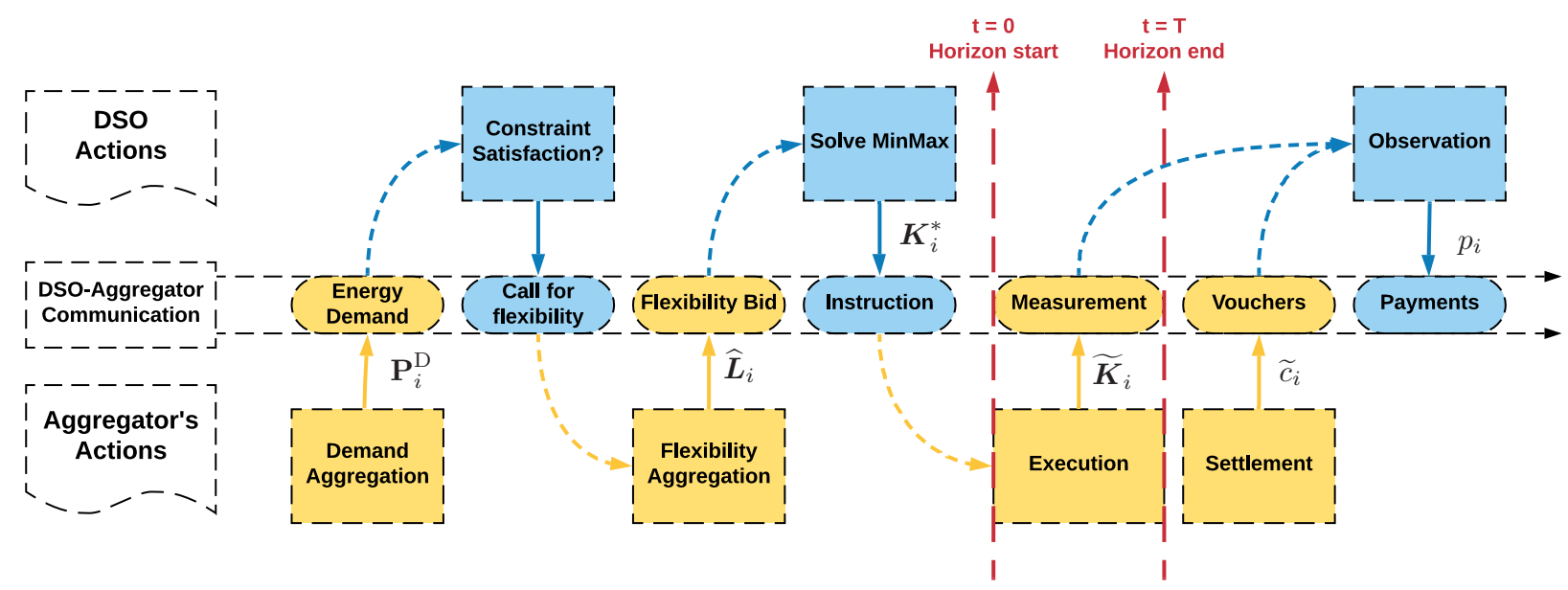

Fig. 1. Procedure and message exchange between DSO and Aggregator

the observed cost for $i$. Parameter $\beta$ is a penalization factor chosen by the DSO.

Let us temporarily assume that all aggregators follow their instruction $\boldsymbol{K}_{i}^{*}$, that is $\widetilde{\boldsymbol{K}}_{i}=\boldsymbol{K}_{i}^{*}$. Then, the CPM mechanism is incentive compatible, and consequently, an implementation of the optimal solution to (11). The intuition behind this is that each aggregator is penalized over the maximum actual flexibility cost of all players. Thus, by making false declarations $\widehat{\boldsymbol{L}}_{i}$, the aggregators can only increase this cost, since a false declaration causes the DSO to allocate flexibility suboptimally. A formal statement and proof follows.

Lemma 1. If the aggregators always follow the instruction, then under the CPM payment rule (13), it is a dominant strategy for each aggregator to declare its true parameters $\widehat{\boldsymbol{L}}_{i}=\boldsymbol{L}_{i}$.

Proof. The proof is provided in Appendix A.

The CPM reward structure was originally designed for task allocation to machines where each machine declares its cost for executing a task. Once the tasks are allocated, the machine has no option but to execute its tasks, and this is why the assumption that the aggregator follows the instruction is needed for the CPM to work in our setting. However, in practice, aggregators are not hard-constrained to follow the instruction. In fact, the aggregator can instead opt for a profile $\widetilde{\boldsymbol{K}}_{i} \neq \boldsymbol{K}_{i}^{*}$. In this case, the original CPM mechanism is not enough to guarantee incentive compatibility. We propose a novel payment rule, called enhanced-CPM (ECPM), modifying the CPM rule as stated by

$$
\begin{array}{r}
p_{i}(\widetilde{\boldsymbol{K}}, \widehat{\boldsymbol{L}})=R_{i}+\widetilde{c}_{i} \\
-\beta \max \left\{\widetilde{c}_{i}, \max _{j \in \Omega_{b}: j \neq i}\left\{c_{j}\left(\boldsymbol{K}_{j}^{*}, \widehat{\boldsymbol{L}}_{j}\right)\right\}\right\} \\
\quad-\sum_{t \in \Omega_{\mathrm{T}}} \gamma\left(\widetilde{K}_{i, t}-K_{i, t}^{*}\right)^{2}
\end{array}
$$

The first term, $R_{i}$, is a fixed payment to each aggregator, in order to make sure that the mechanism is individually rational, i.e., the aggregators' payoff is always positive which makes the aggregators eager to participate in the mechanism rather than opt out. The middle term is (13), while the last term is a penalty controlled by parameter $\gamma$, that has been introduced in order to penalize the aggregator's deviation from the DSO's instruction. The penalty imposed to the deviating aggregator is quadratic in the volume of deviation, so as to penalize deviations in both directions (up and down). Also, the per-unit penalization is increasing in the amount of deviation, which helps towards penalizing large deviations strongly, but keeping the penalty of small (possibly unintended) deviations mild.

The last term is not included in the original CPM mechanism described in [36], but it is necessary in order to incentivize the aggregators to follow the instructions. Regarding the value of $\gamma$, the following theorem states that the proposed mechanism (14) is incentive compatible for $\gamma \gg \lambda_{i, t}$ :

Theorem 1. Given payment structure (14), for $\gamma \gg \lambda_{i, t}$, it is a dominant strategy for each aggregator to declare its true parameters $\left(\widehat{\boldsymbol{L}}_{i}=\boldsymbol{L}_{i}\right)$ and then follow the DSO's instructions $\left(\widetilde{\boldsymbol{K}}_{i}=\boldsymbol{K}_{i}^{*}\right)$.

Proof. The proof is provided in Appendix B.

\section{Remarks and Extensions}

The proposed mechanism of this paper intrinsically assumes a deterministic and perfect information system, where the aggregators have perfect knowledge of their flexibility parameters $\boldsymbol{L}_{i}$. In practice, however, an aggregator may only have a forecast of the parameters that it is required to declare. Thus, there can be a case where an aggregator truthfully declares $\boldsymbol{L}_{i}$ to the best of its abilities, but is then unable to follow the instruction, due to unforeseen changes in the capabilities of its flexibility portfolio and through no fault of its own.

Identifying the reasons behind a deviation from the instruction, and determining whether it occurred due to uncertainty or due to strategic behavior, is a challenging task that requires administrative procedures (e.g. auditing) and falls well beyond the scope of this paper. Nevertheless, against this perspective, ECPM provides the DSO with the ability to adjust the deviation penalty $\gamma$, depending on the system characteristics, so that a good trade-off is achieved between 
not heavily punishing unintended deviations on one hand and disincentivizing strategic behavior on the other.

More generally, the choice of values for parameters $\gamma, R_{i}$ and $\beta$ of ECPM need to be carefully engineered so that the aggregators always benefit from participating in the flexibility market. This means that the penalization for deviations needs to be high enough so as to disincentivize misreporting, but at the same time not so high that aggregators with uncertainties would rather not participate in the market at all, in fear of negative payoffs and heavy penalties. In this context, in Section $\mathrm{V}$, the reader can find examples of suitable choices for parameters $\gamma, R_{i}$ and $\beta$, that address this trade-off in a particular test case.

In view of the uncertainties discussed in this subsection, it is also important to note that the ECPM payment rule (14), also provides resiliency, since an aggregator's payment does not depend on the deviations of others. As a counter-example, consider the simpler payment function

$$
p_{i}=R_{i}+\widetilde{c}_{i}-\beta \max _{n}\left\{\widetilde{c}_{n}\right\}-\sum_{t \in \Omega_{\mathrm{T}}} \gamma\left(\widetilde{K}_{i, t}-K_{i, t}^{*}\right)^{2}
$$

where the term $\max _{n}\left\{\widetilde{c}_{n}\right\}$ is the maximum realized cost over all aggregators (including $i$ ). In a fully deterministic system where aggregators have accurate knowledge over their cost function and are also assumed to follow the instruction, this choice would also align the aggregators' incentives with the DSO's objective. However, in cases of uncertainty in the players' cost parameters $\lambda$, the term $-\max _{n}\left\{\widetilde{c}_{n}\right\}$ of the above choice would unfairly penalize truthful and nondeviating aggregators, due to imperfect $\lambda$ forecasts of other, more volatile, aggregators.

Moreover, such a choice would not only result in unfairness, but would also incentivize an aggregator $i$ to strategically misreport a lower $\lambda_{i, t}$ in order to be allocated more flexibility, so that less flexibility is allocated to another aggregator $j$, which $i$ considers to be volatile. In contrast, in the proposed mechanism, an aggregator's payoff depends only on its own cost realization $\widetilde{c}_{i}$ and not on the cost realizations of others (but only on their declarations, which are proved to be truthful).

Finally, towards extending the system model to include multiple aggregators under the same network node, equations (1) and (2) would read respectively as

$\sum_{k i \in \Omega_{l}} P_{k i, t}-\sum_{i j \in \Omega_{l}}\left(P_{i j, t}+\mathrm{R}_{i j} I_{i j, t}^{\mathrm{sqr}}\right)+P_{i, t}^{\mathrm{S}}=\sum_{a \in \Omega_{\mathrm{A}}^{i}} K_{i, a, t} \mathrm{P}_{i, a, t}^{\mathrm{D}}$

$\sum_{k i \in \Omega_{l}} Q_{k i, t}-\sum_{i j \in \Omega_{l}}\left(Q_{i j, t}+\mathrm{X}_{i j} I_{i j, t}^{\mathrm{sqr}}\right)+Q_{i, t}^{\mathrm{S}}=\sum_{a \in \Omega_{\mathrm{A}}^{i}} K_{i, a, t} \mathrm{Q}_{i, a, t}^{\mathrm{D}}$

where $\Omega_{\mathrm{A}}^{i}$ is the set of aggregators under node $i$ of the network. The ECPM mechanism (and its properties) reads the same as presented, although an aggregator would have to be indexed by $a$, where $a \in \Omega_{\mathrm{A}}^{i}$, rather than simply $i$.

\section{TEst CAses}

Different test cases were performed in order to compare the proposed formulation using a 34-bus medium-voltage, radial, distribution system. System parameters can be found in [37]. The system has 29 load buses each defined by its base load, $\mathrm{P}_{i \text {,base }}$ and $\mathrm{Q}_{i \text {, base }}$. As explained in the introduction, a great deal of flexibility capability stems from small distributed assets, which in turn motivates the need for aggregation. These small assets typically reside at residential or commercial buildings and parking lots. For this reason load buses are assumed in this paper as the system aggregators. Hourly profiles have been shaped by scaling base loads with a loading factor for each timeslot, resembling a typical day-load profile.

\section{A. Assuming truthful declarations of flexibility costs and ac- curate instruction following}

This test aims at showing the difference in the results stemming from the proposed min-max approach, compared to the standard formulation that minimizes the total system cost.

For this particular test, load parameters were randomly selected from normal distributions, i.e., $\mathrm{P}_{i \text {, base }} \sim \mathcal{N}\left(\mu_{P}^{s}, \sigma_{P}^{s}\right)$. Without loss of generality, it was assumed that all aggregators shared the same probability distribution family and a fixed standard deviation of $15 \mathrm{~kW}$. The above setting was simulated for a total of 8 scenarios where $\mu_{P}^{s}=\{100,200, \ldots, 800\} \mathrm{kW}$, and a constant power factor of 0.95 . For each scenario, the results were averaged out over a number of experiments.

Assuming truthful declarations and no inaccuracies, the results of problem (11) were compared to the results of the cost minimization problem

$$
\begin{gathered}
\min _{z, \boldsymbol{K}_{i}, V_{i, t}^{\mathrm{sqr}}, I_{i j, t}^{\mathrm{sqr}}, P_{i j, t}, Q_{i j, t}, P_{0, t}^{\mathrm{S}}, Q_{0, t}^{\mathrm{S}}} \sum_{i \in \Omega_{b}} c_{i}\left(\boldsymbol{K}_{i}, \boldsymbol{L}_{i}\right) \\
\text { (1)-(3), (5)-(8), and (10) }
\end{gathered}
$$

Notice that (17) minimizes the total system cost, whereas the proposed approach minimizes the maximum cost. The total flexibility cost of the system, $\sum_{i \in \Omega_{b}} c_{i}\left(\boldsymbol{K}_{i}^{*}, \boldsymbol{L}_{i}\right)$, is depicted in Fig. 2 for both cases relating the average, maximum, and minimum value of the experiments. It can be seen that the solution to problem (17) minimizes the total cost, as expected. Thus, Fig. 2 can be interpreted as a quantification of the cost of fairness for this particular case study.

Similarly, both approaches were compared in terms of the maximum flexibility cost among aggregators, $\max _{i \in \Omega_{b}} c_{i}\left(\boldsymbol{K}_{i}^{*}, \boldsymbol{L}_{i}\right)$. Results are shown in Fig. 3, again as a function of $\mu_{P}(s)$. Interestingly, the difference is higher in cases where the distribution system is only mildly overloaded.

\section{B. The cost of not knowing the flexibility cost functions}

It is usual for the DSO to receive a flexibility activation service by activating predefined contracts. Those contracts define the aggregator's compensation in a static fashion. Thus, the aggregators do not bid the cost functions $c(\cdot)$, but those are assumed known by the DSO based on the contract. However, the actual flexibility cost of an aggregator is not static and may 


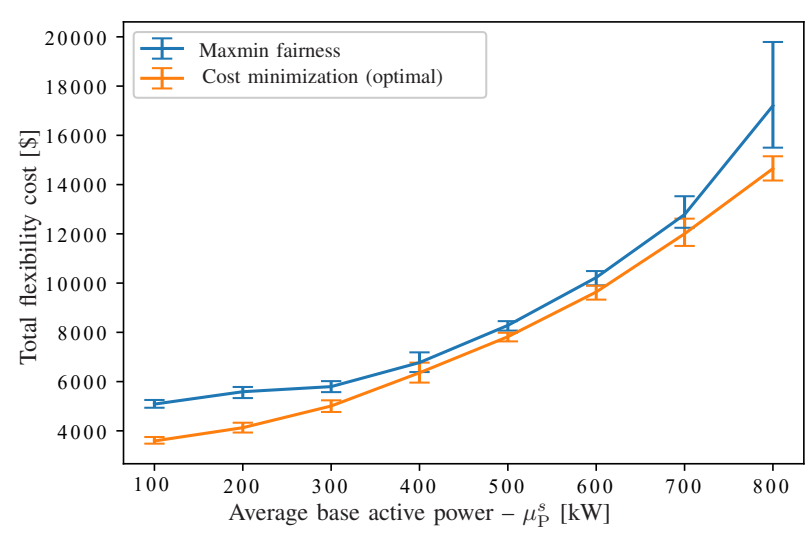

Fig. 2. Cost of fairness: total system cost of the maxmin fair approach compared to the optimal cost

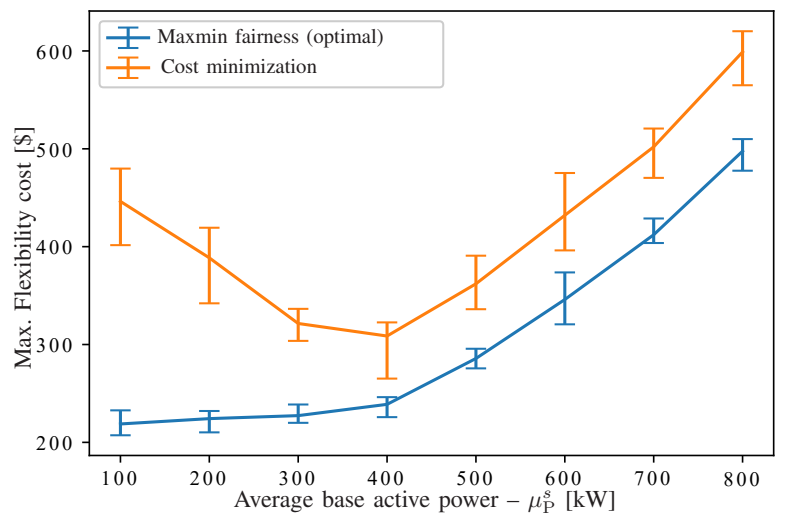

Fig. 3. Maximum flexibility cost among aggregators, for the maxmin fair approach and the cost minimization approach

differ from the assumed costs. In this subsection, we assume that the DSO's estimation of flexibility cost parameters, $\lambda_{i, t}$, is inaccurate. More specifically, for our experiments, the assumed parameter $\widehat{\lambda}_{i, t}$ follows a normal distribution around the actual value $\lambda_{i, t}$, i.e., $\widehat{\lambda}_{i, t} \sim \mathcal{N}\left(\lambda_{i, t}, \sigma_{\lambda}\right)$.

After solving problem (11) using $\widehat{\lambda}_{i, t}$, the DSO determines a suboptimal instruction $\boldsymbol{K}_{i}^{\text {sub }}$. Assuming all aggregators follow the instruction, the actual cost of aggregator $i$ is defined as $c_{i \text {,subopt }}=c_{i}\left(\boldsymbol{K}_{i}^{\mathrm{sub}}, \boldsymbol{L}_{i}\right)$, as in (8).

Thus, the cost of the worst-off aggregator, i.e, the suboptimal value of the objective function in problem (11), is ${ } b j=\max _{i \in \Omega_{b}}\left\{c_{i \text {,subopt }}\right\}$. On the other hand, the optimal objective value of problem (11) is denoted by $o b j^{*}$, hence, $o b j^{*} \leq o b j$. The optimality loss factor caused by inaccurate estimation of the flexibility costs is defined as OptLoss $=o b j / o b j^{*}$ and it is a function of the estimation inaccuracy $\sigma_{\lambda}$. Actual values for $\lambda_{i, t}$ were assumed to follow a normal distribution with average $3\left[\$ / \mathrm{kW}^{2}\right]$ and a standard deviation of $0.5\left[\$ / \mathrm{kW}^{2}\right]$. Results were averaged out over a number of experiments and plotted in Fig. 4 as a function of $\sigma_{\lambda}$.

As observed in Fig. 4, the inefficiency grows with the inaccuracy of the estimated flexibility costs. Note that the inefficiency due to the DSO's inaccurate estimation of $\lambda_{i, t}$ is analog to the case of a false parameter $\widehat{\lambda}_{i, t}$ self-reported by

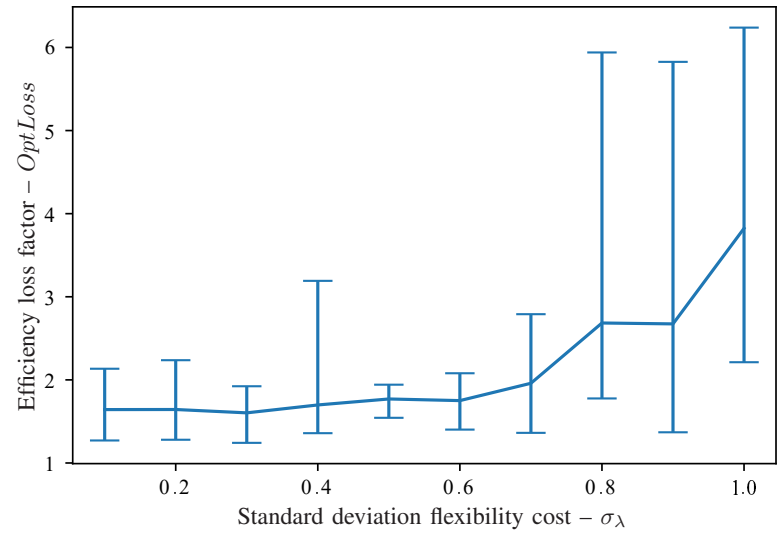

Fig. 4. Optimality loss factor OptLoss as a function of estimation inaccuracy $\sigma_{\lambda}$

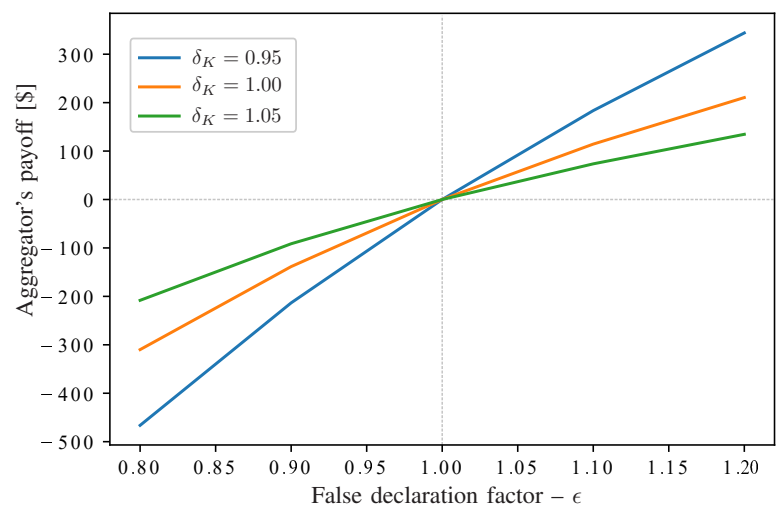

Fig. 5. Aggregator's payoff for the pay-as-bid scheme, as a function of the false declaration factor $\epsilon$ for various cases of instruction deviation factor $\delta_{K}$

the aggregators for strategic reasons. Thus, it is important to incentivize aggregators for truthful reports of their parameters.

\section{Simulating the pay-as-bid scheme and considering inaccu- rate instruction following}

For the next experiment, we take the perspective of a particular aggregator. We assume that the DSO employs the pay-as-bid scheme: In each scenario, the DSO compensates the aggregator by $p_{i}=\widehat{\lambda}_{i, t} \widetilde{K}_{i, t}$, where $\widehat{\lambda}_{i, t}$ is the aggregator's declared flexibility cost. The aggregator's measured flexibility actions $\widetilde{K}_{i, t}$ may differ from the optimal instruction $K_{i, t}^{*}$ by a factor $\delta_{K}$, that is, $\widetilde{K}_{i, t}=\delta_{K} K_{i, t}^{*}$. Assuming a tolerance of $0.05 \%$ in the instruction following, we simulated three scenarios for values of $\delta_{K}=\{0.95,1,1.05\}$.

The aggregator can declare false values $\hat{\lambda}_{i, t}$ on purpose. In the simulation, declaration $\widehat{\lambda}_{i, t}$ was selected as $\widehat{\lambda}_{i, t}=\epsilon \lambda_{i, t}$ where $\epsilon \in[0.8,1.2]$. Note that different values for $\widehat{\lambda}_{i, t}$ results in different instructions for all the aggregators. The results on the aggregator's payoff are depicted in Fig. 5.

It can be seen that the aggregator is able to increase its payoff by declaring a higher flexibility cost than its true one, i.e., $\epsilon>1$. Moreover, by exploiting the tolerance level $\delta_{K}$, the aggregator is able to curtail more load in order to take advantage of the inflated compensation. Thus, it is evident that the pay-as-bid scheme is not suitable for our setting, since 


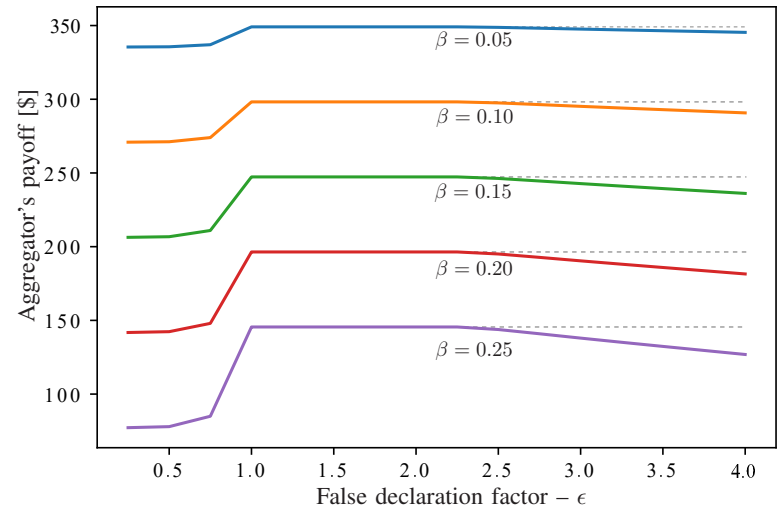

Fig. 6. Aggregator's payoff with ECPM as a function of the deviation $\epsilon$ from the truthful declaration of flexibility costs, for various choices of $\beta$

it might incentivize the aggregators to arbitrarily inflate their bids. This phenomenon has a detrimental effect on the global efficiency, as shown in Section V-B.

\section{Simulating the proposed ECPM scheme with accurate instruction following}

For the next experiment, we assume that the DSO employs the proposed ECPM scheme and that the aggregators perfectly follow the instruction. However, they can still declare false flexibility costs $\hat{\lambda}_{i, t}=\epsilon \lambda_{i, t}$ with $\epsilon \in[0.5,4]$. The aggregator's payoff $\pi_{i}$, given from (39), as a function of $\epsilon$ for different choices of the penalty parameter $\beta$ is depicted in Fig 6. Parameter $R_{i}$ was set to $\$ 400$.

As it can be observed from Fig 6, the aggregator only makes itself worse-off by declaring lower flexibility costs, $\epsilon<1$. The optimal choice would be $\epsilon=1$, which represents a truthful declaration. For $\epsilon>1$, the aggregator inflates its declared flexibility cost, causing the DSO to allocate less flexibility to $i$ and more flexibility to other aggregators. The aggregator's payoff remains unchanged for a range of $\epsilon$ since the DSO can still allocate the necessary flexibility without increasing the term $\max _{j \in \Omega_{b}: j \neq i}\left\{c_{j}\left(\boldsymbol{K}_{j}^{*}, \widehat{\boldsymbol{L}}_{j}\right)\right\}$. However, after a certain point this term also increases, causing the decreasing of the aggregator's payoff.

\section{E. Proof against strategic behavior, using the ECPM scheme}

Finally, we study the case where the aggregator is free to strategically declare its flexibility costs $\widehat{\lambda}_{i, t}=\epsilon \lambda_{i, t}$ and then, employ a flexibility profile $\widetilde{K}_{i, t}=\delta_{K} K_{i, t}^{*}$. The aggregator's payoff $\pi_{i}$, given from (39), was tested using different values for $\beta$ and $\gamma$. The incentives for different aggregators were also assessed. Indicative results are presented below.

In the case that the focal aggregator is not the one with the worst-case cost, then choosing $\epsilon<1$ does not affect its payoff significantly. However, for $\epsilon>1$, (or for $\delta_{K} \neq 1$ ) the aggregator is always worse-off than making a truthful declaration $(\epsilon=1)$ and implementing the instruction $\left(\delta_{K}=1\right)$. This is demonstrated in Fig. 7 and Fig. 8. In case the focal aggregator is the one that is the worst-off, then declaring $\epsilon<1$ also has detrimental effects on its payoff. This is demonstrated in Fig. 9 and Fig. 10.

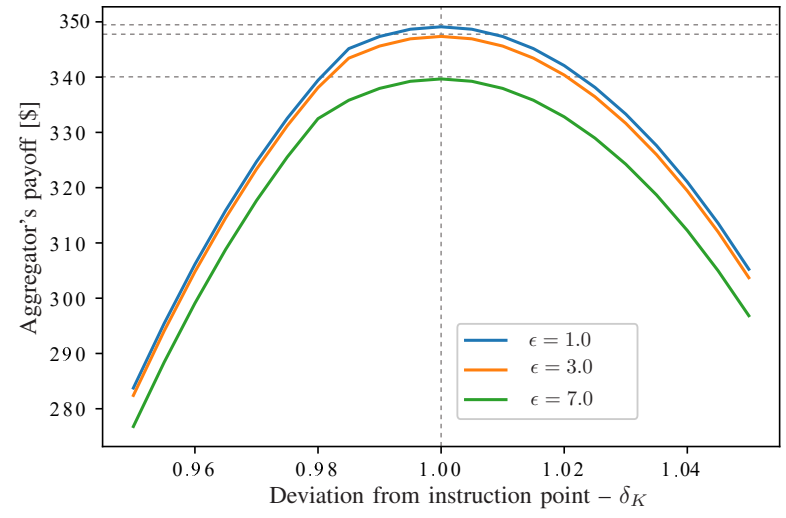

Fig. 7. Aggregator's payoff with ECPM as a function of the deviation $\delta_{K}$ from the instruction for various choices of declaration $\epsilon$, with $\beta=0.05$, $\gamma=1000$.

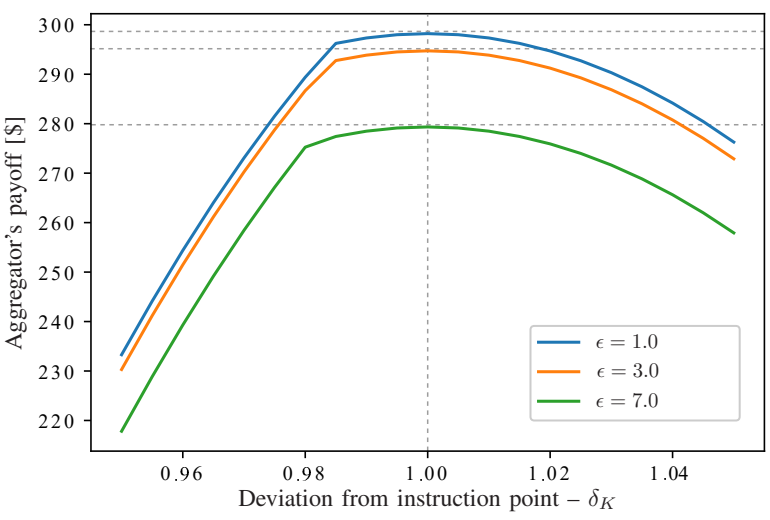

Fig. 8. Aggregator's payoff with ECPM as a function of the deviation $\delta_{K}$ from the instruction for various choices of declaration $\epsilon$, with $\beta=0.1$, $\gamma=500$.

As can be observed, when an aggregator $i$ falsely declares a low flexibility cost $(\epsilon<1)$, then the DSO allocates more flexibility, i.e., lower $K_{i, t}^{*}$ to $i$. Then, $i$ has the incentive to not follow the instruction, and activate less flexibility, i.e., a $\widetilde{K}_{i, t}$ that is closer to $1,(\delta>1)$, in order to decrease its actual flexibility costs and receive a lower penalty from the second factor of the payment function (14). Therefore, as can be seen for all tested cases, the aggregator's payoff-maximizing strategy is to make a truthful declaration $(\epsilon=1)$ and then perfectly follow the instruction given by the $\operatorname{DSO}(\delta=1)$.

\section{CONCLUSIONS AND FUTURE WORK}

In this paper, we formulated the problem of satisfying the constraints of the distribution system in a max-min fair way, through a flexibility market for aggregators. We provided an incentive compatible mechanism, so that aggregators truthfully declare their flexibility costs and then follow the DSO's instructions. Without such a mechanism, aggregators would inflate their bids, which results in detrimental effects on the system's efficiency. In particular, our simulations show that without accounting for truthful declarations, the cost of the worst-off player in the system can grow by a factor of up to four times, compared to the optimal min-max allocation. The property of incentive compatibility was theoretically proven, 


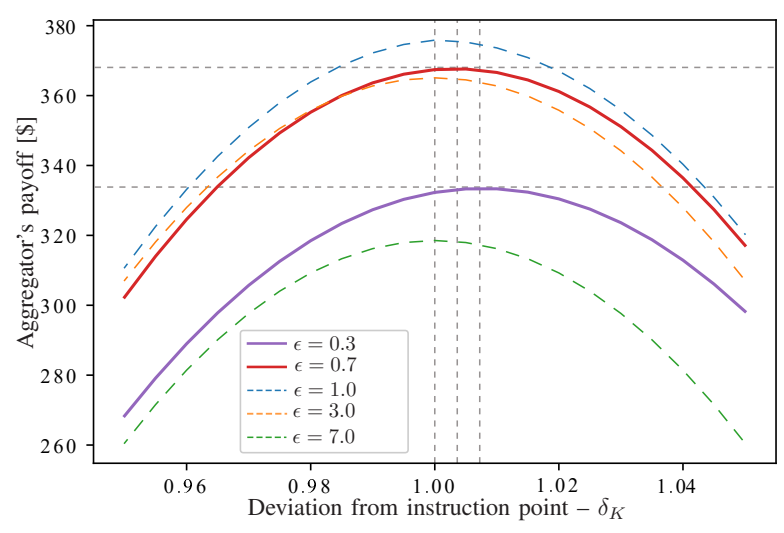

Fig. 9. Aggregator's payoff with ECPM as a function of the deviation $\delta_{K}$ from the instruction for various choices of declaration $\epsilon$, with $\beta=0.02$, $\gamma=1000$

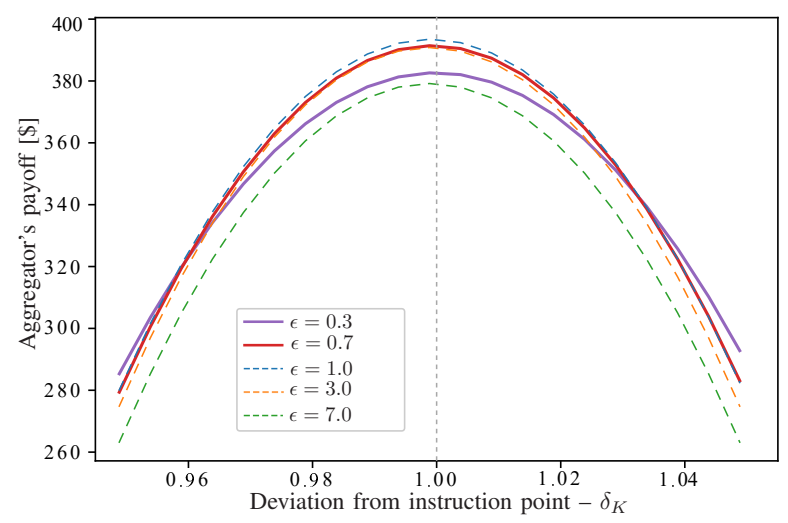

Fig. 10. Aggregator's payoff with ECPM as a function of the deviation $\delta_{K}$ from the instruction for various choices of declaration $\epsilon$, with $\beta=0.005$, $\gamma=2000$

while the system's behavior was tested in a number of scenarios. In these scenarios, we are able to show that the proposed mechanism can be fine-tuned effectively, so that aggregators are not heavily penalized for small deviations that can occur in practice, while at the same time the property of incentivizing truthful declarations is preserved.

Future work can include a detailed modelling of the aggregators' flexible assets, while also extend the proposed scheme to be iteratively executed (in a rolling horizon fashion) and maintain the property of incentive compatibility. Moreover, future work can study the properties of incentive compatibility and optimality in cases of multi-objective optimization, e.g., when the operator pursues a combination of social welfare maximization and max-min fairness.

\section{APPENDIX A}

\section{PROOF OF LEMMA 1}

With payment rule (13), the aggregator's payoff $\pi_{i}$ from (12), considering $\widetilde{\boldsymbol{K}}_{i}=\boldsymbol{K}_{i}^{*}$ is given by

$$
\pi_{i}=-\beta \max \left\{\widetilde{c}_{i}, \max _{j \in \Omega_{b}: j \neq i}\left\{c_{j}\left(\boldsymbol{K}_{j}^{*}, \widehat{\boldsymbol{L}}_{j}\right)\right\}\right\}
$$

Thus, the aggregator's penalty is over its actual flexibility cost, or over the maximum declared flexibility cost of other players, whichever is highest. Let $c_{i}^{*}=c_{i}\left(\boldsymbol{K}_{i}^{*}, \boldsymbol{L}_{i}\right)$ denote the cost of player $i$ at the instruction $\boldsymbol{K}_{i}^{*}$ which resulted by (11), under truthful declarations. Also, let $\boldsymbol{K}_{i}^{\text {sub }}$ denote the instruction of $i$ under a non-truthful declaration from any of the players. Finally, let $c_{i}\left(\boldsymbol{K}_{i}^{\mathrm{sub}}\right)$ denote the actual cost of player $i$ under non-truthful declarations. With ties broken arbitrarily, we have the following two cases:

- under truthful declarations, $i$ is not the player with the highest flexibility cost at the instructions point i.e.

$$
c_{i}^{*}<\max _{j \in \Omega_{b}: j \neq i}\left\{c_{j}^{*}\right\}
$$

and also

$$
\max _{j \in \Omega_{b}: j \neq i}\left\{c_{j}^{*}\right\}=\max _{n \in \Omega_{b}}\left\{c_{n}^{*}\right\}
$$

By (18), $i$ 's payoff under truthful declarations is

$$
\pi_{i}=-\beta \max _{j \in \Omega_{b}: j \neq i}\left\{c_{j}^{*}\right\}
$$

Let us assume that $i$ declares a higher flexibility cost $\left(\widehat{\lambda}_{i, t}>\lambda_{i, t}\right.$, and/or $\underline{\widehat{\mathrm{K}}}_{i, t}>\underline{\mathrm{K}}_{i, t}$ and/or $\left.\widehat{\overline{\mathrm{K}}}_{i, t}<\overline{\mathrm{K}}_{i, t}\right)$. Then, it would cause the DSO to allocate more flexibility to other players $j$, which can only increase their flexibility cost, i.e.

$$
\max _{j \in \Omega_{b}: j \neq i}\left\{c_{j}\left(\boldsymbol{K}_{j}^{\mathrm{sub}}\right)\right\}>\max _{j \in \Omega_{b}: j \neq i}\left\{c_{j}^{*}\right\}
$$

There are two subcases:

$-i$ is still not the player with the highest flexibility cost at the new instructions point

$$
c_{i}\left(\boldsymbol{K}_{i}^{\mathrm{sub}}\right)<\max _{j \in \Omega_{b}: j \neq i}\left\{c_{j}\left(\boldsymbol{K}_{j}^{\mathrm{sub}}\right)\right\}
$$

In this subcase $i$ 's payoff is equal to $-\beta \max _{j \in \Omega_{b}: j \neq i}\left\{c_{j}\left(\boldsymbol{K}_{j}^{\text {sub }}\right)\right\}$, which, from (22) and (21), is lower than $i$ 's payoff under truthful declarations.

- i's flexibility cost at the new instruction point is the highest

$$
c_{i}\left(\boldsymbol{K}_{i}^{\mathrm{sub}}\right)=\max _{n \in \Omega_{b}}\left\{c_{n}\left(\boldsymbol{K}_{n}^{\mathrm{sub}}\right)\right\}
$$

In this subcase $i$ 's payoff is equal to $-\beta c_{i}\left(\boldsymbol{K}_{i}^{\mathrm{sub}}\right)$. For $i$ to be better-off, it needs to be:

$$
c_{i}\left(\boldsymbol{K}_{i}^{\mathrm{sub}}\right)<\max _{j \in \Omega_{b}: j \neq i}\left\{c_{j}^{*}\right\}
$$

which, by (24) and (20), gives

$$
\max _{n \in \Omega_{b}}\left\{c_{n}\left(\boldsymbol{K}_{n}^{\text {sub }}\right)\right\}<\max _{n \in \Omega_{b}}\left\{c_{n}^{*}\right\}
$$

However, this is not possible because it contradicts the fact that problem (11) provides the optimal solution, i.e. the one that minimizes the maximum cost.

Now let us assume that $i$ declares a lower flexibility cost $\left(\widehat{\lambda}_{i, t}<\lambda_{i, t}\right.$, and/or $\widehat{\widehat{K}}_{i, t}<\underline{\mathrm{K}}_{i, t}$ and/or $\left.\widehat{\overline{\mathrm{K}}}_{i, t}>\overline{\mathrm{K}}_{i, t}\right)$. Then, the DSO allocates more flexibility to $i$ and less to other players, which means that

$$
\max _{j \in \Omega_{b}: j \neq i}\left\{c_{j}\left(\boldsymbol{K}_{j}^{\mathrm{sub}}\right)\right\} \leq \max _{j \in \Omega_{b}: j \neq i}\left\{c_{j}^{*}\right\}
$$

There are two subcases: 
- $i$ is still not the player with the highest flexibility cost at the new instructions point, as in (23). In this subcase $i$ 's payoff is equal to $-\beta \max _{j \in \Omega_{b}: j \neq i}\left\{c_{j}\left(\boldsymbol{K}_{j}^{\text {sub }}\right)\right\}$. For $i$ to be better-off, it needs to be

$$
\max _{n \in \Omega_{b}}\left\{c_{n}\left(\boldsymbol{K}_{n}^{\mathrm{sub}}\right)\right\}<\max _{n \in \Omega_{b}}\left\{c_{n}^{*}\right\}
$$

However, since problem (11) provides the optimal solution that minimizes $\max _{n \in \Omega_{b}}\left\{c_{n}^{*}\right\}$, eq. (28) could only stand if

$$
\boldsymbol{K}_{j}^{\text {sub }}<\underline{\mathbf{K}}_{i, t}
$$

or

$$
\boldsymbol{K}_{j}^{\mathrm{sub}}>\overline{\mathrm{K}}_{i, t}
$$

either of which would constitute $\boldsymbol{K}_{j}^{\text {sub }}$ an infeasible solution to problem (11). Therefore, it would not be possible for the aggregator to follow the instruction, which, in turn, contradicts the assumption of Lemma 1.

- $i$ 's flexibility cost at the new instruction point is the highest, as in (24). This can be equivalently written as

$$
c_{i}\left(\boldsymbol{K}_{i}^{\mathrm{sub}}\right)=\max _{n \in \Omega_{b}}\left\{c_{n}\left(\boldsymbol{K}_{n}^{\mathrm{sub}}\right)\right\}
$$

In this subcase, $i$ 's payoff is equal to $-\beta c_{i}\left(\boldsymbol{K}_{i}^{\mathrm{sub}}\right)$. For $i$ to be better-off, it needs to be as in (25). Because of (19), this means that

$$
c_{i}\left(\boldsymbol{K}_{i}^{\mathrm{sub}}\right)<\max _{n \in \Omega_{b}}\left\{c_{n}^{*}\right\}
$$

which, by (31), becomes equivalent to (28) and thus contradictory by the same argument made in the previous subcase.

- under truthful declarations, $i$ is the player with the highest flexibility cost at the instructions point i.e.

$$
c_{i}^{*}>\max _{j \in \Omega_{b}: j \neq i}\left\{c_{j}^{*}\right\}
$$

In this case, if $i$ declares a lower flexibility cost, then it would cause the DSO to allocate more flexibility to $i$. This would increase $i$ 's flexibility cost i.e. $c_{i}\left(\boldsymbol{K}_{i}^{\text {sub }}\right)>c_{i}^{*}$. However, by (18), $i$ 's payoff is less than or equal to $-\beta c_{i}\left(\boldsymbol{K}_{i}^{\mathrm{sub}}\right)$. Thus, $i$ can only be made worse-off by increasing $c_{i}\left(\boldsymbol{K}_{i}^{\text {sub }}\right)$ in this case.

On the other hand, if $i$ declares a higher flexibility cost, the DSO allocates less flexibility to $i$. Again, there are two subcases:

- $i$ is still the player with the highest flexibility cost at the new instructions point, i.e., $c_{i}\left(\boldsymbol{K}_{i}^{\text {sub }}\right)>\max _{j \in \Omega_{b}: j \neq i}\left\{c_{j}\left(\boldsymbol{K}_{j}^{\text {sub }}\right)\right\}$. For $i$ to be better-off, it has to be

$$
c_{i}\left(\boldsymbol{K}_{i}^{\mathrm{sub}}\right)<c_{i}^{*}
$$

Since, by assumption of this subcase, $i$ is the player with the highest flexibility cost under both truthful and non-truthful declarations, we have that $c_{i}\left(\boldsymbol{K}_{i}^{\text {sub }}\right)=$ $\max _{n \in \Omega_{b}}\left\{c_{n}\left(\boldsymbol{K}_{n}^{\mathrm{sub}}\right)\right\}$ and $c_{i}^{*}=\max _{n \in \Omega_{b}}\left\{c_{n}^{*}\right\}$. Thus, eq. (34) becomes equivalent to (26), which contradicts the fact that $\max _{n \in \Omega_{b}}\left\{c_{n}^{*}\right\}$ is the optimal solution to (11).
- $i$ is no longer the player with the highest flexibility cost i.e.

$$
c_{i}\left(\boldsymbol{K}_{i}^{\mathrm{sub}}\right)<\max _{j \in \Omega_{b}: j \neq i}\left\{c_{j}\left(\boldsymbol{K}_{j}^{\mathrm{sub}}\right)\right\}
$$

or, equivalently

$$
\max _{j \in \Omega_{b}: j \neq i}\left\{c_{j}\left(\boldsymbol{K}_{j}^{\mathrm{sub}}\right)\right\}=\max _{n \in \Omega_{b}}\left\{c_{n}\left(\boldsymbol{K}_{n}^{\mathrm{sub}}\right)\right\}
$$

Similarly, (33) can also be written as

$$
c_{i}^{*}=\max _{n \in \Omega_{b}}\left\{c_{n}^{*}\right\}
$$

For $i$ to be better-off, it needs to be

$$
\max _{j \in \Omega_{b}: j \neq i}\left\{c_{j}\left(\boldsymbol{K}_{j}^{\mathrm{sub}}\right)\right\}<c_{i}^{*}
$$

which, by (36) and (37), is equivalent to (26), and therefore contradictory.

Thus, the aggregator cannot benefit from false declarations.

\section{APPENDIX B}

\section{PROOF OF THEOREM 1}

With payment structure (14), the aggregator's payoff is

$$
\begin{aligned}
\pi_{i}=R_{i}-\beta \max \left\{\widetilde{c}_{i}, \max _{j \in \Omega_{b}: j \neq i}\left\{c_{j}\left(\boldsymbol{K}_{j}^{*}, \widehat{\boldsymbol{L}}_{j}\right)\right\}\right\} \\
-\sum_{t \in \Omega_{\mathrm{T}}} \gamma\left(\widetilde{K}_{i, t}-K_{i, t}^{*}\right)^{2}
\end{aligned}
$$

Consider the aggregator's problem of deciding on the actual flexibility profile $\widetilde{\boldsymbol{K}}_{i}$. Parameter $R_{i}$ and term $\max _{j \in \Omega_{b}: j \neq i}\left\{c_{j}\left(\boldsymbol{K}_{j}^{*}, \widehat{\boldsymbol{L}}_{j}\right)\right\}$ are fixed numbers. The aggregator's payoff optimization problem reads as

$$
\begin{aligned}
\min _{\boldsymbol{K}_{j}^{*}, \widehat{\boldsymbol{L}}_{j}}\left\{\max \left\{\widetilde{c}_{i}, \max _{j \in \Omega_{b}: j \neq i}\left\{c_{j}\left(\boldsymbol{K}_{j}^{*}, \widehat{\boldsymbol{L}}_{j}\right)\right\}\right\}\right. \\
\left.+\sum_{t \in \Omega_{\mathrm{T}}} \gamma\left(\widetilde{K}_{i, t}-K_{i, t}^{*}\right)^{2}\right\}
\end{aligned}
$$

After introducing slack variable $\zeta$, (40) is rewritten as

$$
\begin{aligned}
\min _{\boldsymbol{K}_{j}^{*}, \widehat{\boldsymbol{L}}_{j}} & \zeta+\sum_{t \in \Omega_{\mathrm{T}}} \gamma\left(\widetilde{K}_{i, t}-K_{i, t}^{*}\right)^{2} \\
\zeta & \geq \max _{j \in \Omega_{b}: j \neq i}\left\{c_{j}\left(\boldsymbol{K}_{j}^{*}, \widehat{\boldsymbol{L}}_{j}\right)\right\} \\
\zeta & \geq \widetilde{c}_{i}
\end{aligned}
$$

The worst possible case happens when $\max _{j \in \Omega_{b}: j \neq i}\left\{c_{j}\left(\boldsymbol{K}_{j}^{*}, \widehat{\boldsymbol{L}}_{j}\right)\right\}=0$, since in this case the aggregator has maximum freedom to minimize its penalty. Hence, since $\widetilde{c}_{i} \geq 0$, we have $\min _{\widetilde{K}_{i, t}}\left\{f\left(\widetilde{K}_{i, t}\right)\right\}$, where

$$
f\left(\widetilde{K}_{i, t}\right)=\left\{\widetilde{c}_{i}+\sum_{t \in \Omega_{\mathrm{T}}} \gamma\left(\widetilde{K}_{i, t}-K_{i, t}^{*}\right)^{2}\right\}
$$

By taking the stationary point of (41) for $\widetilde{K}_{i, t}$, we have

$$
\widetilde{K}_{i, t}=\frac{\lambda_{i, t}+\gamma K_{i, t}^{*}}{\lambda_{i, t}+\gamma}
$$

for $\gamma \gg \lambda_{i, t}$, we have $\widetilde{K}_{i, t} \approx K_{i, t}^{*}$. Thus, the aggregator is incentivized to follow the instruction, which, combined with Lemma 1, completes the proof. 


\section{ACKNOWLEDGEMENT}

We would like to thank the anonymous reviewers for their valuable comments and corrections that improved the quality of the manuscript. Especially, we are indebted to one of the reviewers who helped in producing a better version of the proof for Lemma 1.

\section{REFERENCES}

[1] L. Gkatzikis, I. Koutsopoulos, and T. Salonidis, "The role of aggregators in smart grid demand response markets," IEEE Journal on Selected Areas in Communications, vol. 31, no. 7, pp. 1247-1257, 2013.

[2] G. Tsaousoglou, P. Makris, and E. Varvarigos, "Electricity market policies for penalizing volatility and scheduling strategies: The value of aggregation, flexibility, and correlation," Sust. Energy, Grids and Networks (SEGAN), vol. 12, pp. 57-68, December 2017.

[3] X. Chen, E. Dall'Anese, C. Zhao, and N. Li, "Aggregate power flexibility in unbalanced distribution systems," IEEE Transactions on Smart Grid, vol. 11, no. 1, pp. 258-269, 2020.

[4] S. H. Low, "Convex relaxation of optimal power flow-part ii: Exactness," IEEE Transactions on Control of Network Systems, vol. 1, no. 2, pp. 177-189, Jun. 2014

[5] S. Huang, Q. Wu, J. Wang, and H. Zhao, "A sufficient condition on convex relaxation of AC optimal power flow in distribution networks," IEEE Transactions on Power Systems, vol. 32, no. 2, pp. 1359-1368, Mar. 2017.

[6] S. Vandael, B. Claessens, M. Hommelberg, T. Holvoet, and G. Deconinck, "A scalable three-step approach for demand side management of plug-in hybrid vehicles," IEEE Transactions on Smart Grid, vol. 4, no. 2, pp. 720-728, 2013.

[7] G. Tsaousoglou, K. Steriotis, and E. Varvarigos, "A stochastic approximation method for price-based assignment of electric vehicles to charging stations," in 2019 International Conference on Smart Energy Systems and Technologies (SEST), 2019, pp. 1-6.

[8] J. Saez-Gallego, J. M. Morales, M. Zugno, and H. Madsen, "A datadriven bidding model for a cluster of price-responsive consumers of electricity," IEEE Transactions on Power Systems, vol. 31, no. 6, pp. 5001-5011, 2016

[9] G. Tsaousoglou, K. Steriotis, N. Efthymiopoulos, K. Smpoukis, and E. Varvarigos, "Near-optimal demand side management for retail electricity markets with strategic users and coupling constraints," Sustainable Energy, Grids and Networks, vol. 19, 2019.

[10] J. Guerrero, D. Gebbran, S. Mhanna, A. C. Chapman, and G. Verbič, "Towards a transactive energy system for integration of distributed energy resources: Home energy management, distributed optimal power flow, and peer-to-peer energy trading," Renewable and Sustainable Energy Reviews, vol. 132, 2020.

[11] E. G. Kardakos, C. K. Simoglou, and A. G. Bakirtzis, "Optimal offering strategy of a virtual power plant: A stochastic bi-level approach," IEEE Transactions on Smart Grid, vol. 7, no. 2, pp. 794-806, 2016.

[12] M. Yazdani-Damavandi, N. Neyestani, M. Shafie-khah, J. Contreras, and J. P. S. Catalão, "Strategic behavior of multi-energy players in electricity markets as aggregators of demand side resources using a bilevel approach," IEEE Transactions on Power Systems, vol. 33, no. 1, pp. 397-411, 2018.

[13] K. Steriotis, K. Smpoukis, N. Efthymiopoulos, G. Tsaousoglou, P. Makris, and E. Varvarigos, "Strategic and network-aware bidding policy for electric utilities through the optimal orchestration of a virtual and heterogeneous flexibility assets' portfolio," Electric Power Systems Research, vol. 184, 2020.

[14] M. G. Vayá, L. B. Roselló, and G. Andersson, "Optimal bidding of plug-in electric vehicles in a market-based control setup," in 2014 Power Systems Computation Conference, 2014, pp. 1-8.

[15] L. Bai, J. Wang, C. Wang, C. Chen, and F. Li, "Distribution locational marginal pricing (DLMP) for congestion management and voltage support," IEEE Transactions on Power Systems, vol. 33, no. 4, pp. 40614073,2018

[16] S. Huang, Q. Wu, S. S. Oren, R. Li, and Z. Liu, "Distribution locational marginal pricing through quadratic programming for congestion management in distribution networks," IEEE Transactions on Power Systems, vol. 30, no. 4, pp. 2170-2178, 2015.

[17] P. Jacquot, "DLMP-based coordination procedure for decentralized demand response under distribution network constraints," arXiv, math.OC, 2020.
[18] C. Lin, W. Wu, and M. Shahidehpour, "Decentralized AC optimal power flow for integrated transmission and distribution grids," IEEE Transactions on Smart Grid, vol. 11, no. 3, pp. 2531-2540, 2020.

[19] W. Liu, Q. Wu, F. Wen, and J. Østergaard, "Day-ahead congestion management in distribution systems through household demand response and distribution congestion prices," IEEE Transactions on Smart Grid, vol. 5, no. 6, pp. 2739-2747, 2014.

[20] A. Perez-Diaz, E. Gerding, and F. McGroarty, "Catching cheats: Detecting strategic manipulation in distributed optimisation of electric vehicle aggregators," Journal of Artificial Intelligence Research, vol. 67, 2019.

[21] H. Rose, A. Rogers, and E. H. Gerding, "A scoring rule-based mechanism for aggregate demand prediction in the smart grid," in Proceedings of the 11th International Conference on Autonomous Agents and Multiagent Systems - Volume 2, ser. AAMAS '12. International Foundation for Autonomous Agents and Multiagent Systems, 2012, p. 661-668.

[22] C. Akasiadis and G. Chalkiadakis, "Agent cooperatives for effective power consumption shifting," in Proceedings of the Twenty-Seventh AAAI Conference on Artificial Intelligence, ser. AAAI'13. AAAI Press, 2013, p. 1263-1269.

[23] S. Mhanna, A. C. Chapman, and G. Verbič, "A faithful and tractable distributed mechanism for residential electricity pricing," IEEE Transactions on Power Systems, vol. 33, no. 4, pp. 4238-4252, 2018.

[24] L. Exizidis, J. Kazempour, A. Papakonstantinou, P. Pinson, Z. De Grève, and F. Vallée, "Incentive-compatibility in a two-stage stochastic electricity market with high wind power penetration," IEEE Transactions on Power Systems, vol. 34, no. 4, pp. 2846-2858, 2019.

[25] P. Samadi, H. Mohsenian-Rad, R. Schober, and V. W. S. Wong, "Advanced demand side management for the future smart grid using mechanism design," IEEE Transactions on Smart Grid, vol. 3, no. 3, pp. $1170-1180,2012$.

[26] G. Tsaousoglou, K. Steriotis, N. Efthymiopoulos, P. Makris, and E. Varvarigos, "Truthful, practical and privacy-aware demand response in the smart grid via a distributed and optimal mechanism," IEEE Transactions on Smart Grid, pp. 1-1, 2020.

[27] Z. Baharlouei, M. Hashemi, H. Narimani, and H. Mohsenian-Rad, "Achieving optimality and fairness in autonomous demand response: Benchmarks and billing mechanisms," IEEE Transactions on Smart Grid, vol. 4, no. 2, pp. 968-975, 2013

[28] N. Yaagoubi and H. T. Mouftah, "Fairness-aware game theoretic approach for demand response in microgrids," in 2015 Seventh Annual IEEE Green Technologies Conference, 2015, pp. 125-131.

[29] C. Kok, J. Kazempour, and P. Pinson, "A DSO-level contract market for conditional demand response," in 2019 IEEE PowerTech, 2019, pp. 1-6.

[30] K. Steriotis, G. Tsaousoglou, N. Efthymiopoulos, P. Makris, and E. Varvarigos, "A novel behavioral real time pricing scheme for the active energy consumers' participation in emerging flexibility markets," Sust. Energy, Grids and Networks (SEGAN), vol. 16, pp. $14-27,2018$.

[31] G. Tsaousoglou, N. Efthymiopoulos, P. Makris, and E. Varvarigos, "Personalized real time pricing for efficient and fair demand response in energy cooperatives and highly competitive flexibility markets," Jour. of Modern Power Syst. and Clean Energy, vol. 7, pp. 151 - 162, 2019.

[32] D. Nace and M. Pioro, "Max-min fairness and its applications to routing and load-balancing in communication networks: a tutorial," IEEE Communications Surveys Tutorials, vol. 10, no. 4, pp. 5-17, 2008.

[33] G. Tsaousoglou, P. Pinson, and N. G. Paterakis, "Max-min fairness for demand side management under high RES penetration: Dealing with undefined consumer valuation functions," in 2020 International Conference on Smart Energy Systems and Technologies (SEST), 2020, pp. $1-6$.

[34] F. Moret and P. Pinson, "Energy collectives: A community and fairness based approach to future electricity markets," IEEE Transactions on Power Systems, vol. 34, no. 5, pp. 3994-4004, 2019.

[35] J. S. Giraldo, J. A. Castrillon, J. C. López, M. J. Rider, and C. A. Castro, "Microgrids energy management using robust convex programming," IEEE Transactions on Smart Grid, vol. 10, no. 4, pp. 4520-4530, 2019.

[36] Y. Shoham and K. Leyton-Brown, "Compensation and penalty mechanisms," in Multiagent Systems: Algorithmic, Game-Theoretic, and Logical Foundations. Cambridge University Press, 2009.

[37] U. Laboratorio de Planejamento de Sistemas de Energia Eletrica (LaPSEE), "Downloads / sistemas testes," https://www. feis.unesp.br/departamentos/engenharia-eletrica/pesquisas-e-projetos/ lapsee/downloads/materiais-de-cursos1193/, 2020. 